\title{
The accuracy of MRI in diagnosing and classifying acute traumatic multiple ligament knee injuries
}

\author{
Xusheng $\mathrm{Li}^{1 \dagger}$, Qian Hou ${ }^{1 \dagger}$, Xuehua Zhan ${ }^{1}$, Long Chang ${ }^{1}$, Xiaobing $\mathrm{Ma}^{2}$ and Haifeng Yuan ${ }^{1 *}$
}

\begin{abstract}
Background: Magnetic resonance imaging (MRI) is widely used for the evaluation of knee injuries, however, the accuracy of MRI in classifying multiple ligament knee injuries (MLKIs) remains unknown. This study aimed to investigate the accuracy of MRI in diagnosing and classifying acute traumatic MLKIs, we hypothesize that MRI had high accuracy in detecting and classifying MLKIs.

Methods: The clinical data of 97 patients who were diagnosed with acute traumatic MLKIs and managed by multiligament reconstruction between 2012 and 2020 were retrospectively reviewed. The MR images were read by two experienced radiologists and results were compared with intraoperative findings, which were considered as the reference for the identification of injured structures. The value of MRI in detecting injuries of anterior cruciate ligament $(\mathrm{ACL})$, posterior cruciate ligament $(\mathrm{PCL})$, medial collateral ligament $(\mathrm{MCL})$, lateral collateral ligament $(\mathrm{LCL})$, and meniscus was evaluated by calculating the sensitivity, specificity, positive predictive value, negative predictive value, positive likelihood ratio, negative likelihood ratio, and kappa coefficients analysis. The value of MRI in classifying MLKIs was evaluated by calculating the agreement between MRI and intraoperative findings.
\end{abstract}

Results: For detecting the specific injured structures in MLKIs, MRI had high sensitivity $(90.7 \%$ for $A C L, 90.4 \%$ for PCL, and moderate specificity ( $63.6 \%$ for $\mathrm{ACL}, 50 \%$ for $\mathrm{PCL}$ ) in detecting cruciate ligament injuries, moderate sensitivity (79.1\% for MCL, 55.6\% for $\mathrm{LCL}$ ) and specificity (46.7\% for MCL, 68.4\% for $\mathrm{LCL}$ ) in detecting collateral ligament injuries, fair sensitivity (61.5\%) and low specificity (39.4\%) in the diagnosis of injuries to the meniscus. For classifying the MIKIs, MRI had a moderate agreement with intraoperative findings in classifying KD-V (kappa value $=0.57$ ), poor agreement in the KD-I (kappa value $=0.39$ ) and KD-IIIM (kappa value = 0.31), meaningless in the KD-II and KD-IIIL (kappa value $<0$ ). The overall agreement between MRI and intraoperative findings in classifying MLKIs was poor (kappa value $=0.23)$.

Conclusions: MRI is valuable in early detection and diagnosis of acute MLKIs, however, the accuracy of MRI in classifying MLKIs is limited. The management of MLKIs should be based on intraoperative findings, physical examinations, and comprehensive imaging results.

Keywords: Multiple ligaments injuries, Knee dislocations, MRI, Value

*Correspondence: yuanhaifeng000@126.com

${ }^{\dagger}$ Xusheng Li and Qian Hou contributed equally to this work.

${ }^{1}$ Department of Spinal Orthopaedics, General Hospital of Ningxia Medical University, No. of 804, Shengli Street, Yinchuan 750004, China

Full list of author information is available at the end of the article

\section{Introduction}

Multi-ligament knee injuries (MLKIs) are rare but serious injuries that are usually caused by high-energy trauma [1-3]. The definition of MLKIs is the complete tear of 2 or more cruciate and/or collateral ligaments, with or without injuries of meniscus, nerves, arteries, original author(s) and the source, provide a link to the Creative Commons licence, and indicate if changes were made. The images or other third party material in this article are included in the article's Creative Commons licence, unless indicated otherwise in a credit line to the material. If material is not included in the article's Creative Commons licence and your intended use is not permitted by statutory regulation or exceeds the permitted use, you will need to obtain permission directly from the copyright holder. To view a copy of this licence, visit http://creativecommons.org/licenses/by/4.0/. The Creative Commons Public Domain Dedication waiver (http://creativeco mmons.org/publicdomain/zero/1.0/) applies to the data made available in this article, unless otherwise stated in a credit line to the data. 
or periarticular fractures [4]. Some of the MLKIs have knee dislocations (KD), however, the dislocated knee can reduce spontaneously or have been reduced in the emergency department before hospitalization, thus the severity of the injured knee can be underestimated $[1,5,6]$.

Early detection of injured structures is crucial for the management of MLKIs, MRI is the necessary preoperative imaging examination, which is also valuable in detecting nerve injuries [7]. The value of MRI for diagnosing isolated ligament injuries has been widely demonstrated, however, in terms of multi-ligament injuries, the accuracy of MRI is controversial. Derby et al. [8] found that MRI was sensitive in detecting injuries of cruciate and collateral ligaments, but not reliable in diagnosing injury to the meniscus or posterolateral corner (PLC). Twaddle et al. [9] demonstrated that MRI is not reliable for revealing injuries of the lateral collateral ligament (LCL) and PLC. However, Munshi et al. [10] reported that MRI had reliable sensitivity and specificity for detecting cruciate ligament injury and meniscal tears, even injuries that could not be precisely identified by arthroscopy. Similar results were found by Halinen et al. [11], and Kosy et al. [12]. In terms of reproducibility, Barbier et al. [13] demonstrated that MRI lacks precision and reproducibility, and the diagnosis should be integrated with clinical exam and stress $\mathrm{X}$-rays. It has been also reported that MRI was inferior to clinical examination [14].

In short, the diagnostic value of MRI in MLKIs remains controversial, and the accuracy of MRI in classifying MLKIs has not been reported. This study aimed to investigate the accuracy of MRI in diagnosing and classifying acute traumatic MLKIs (within 3 weeks after injury). The intra-operative findings were considered the reference of injury patterns. We hypothesize that MRI had high accuracy in detecting and classifying MLKIs.

\section{Methods \\ Patients}

The clinical record database of knee surgeries in the orthopaedic department from one single center between 2012 and 2020 was retrospectively reviewed. Patients who were diagnosed with MLKIs and treated by multiligament reconstruction were included. The inclusion criteria were (1) Acute traumatic injury of at least two of the following ligaments: ACL, PCL, MCL, and LCL. (2) The 1.5 Tesla MRI was performed preoperatively and the images were available. (3) The injury patterns of knee structures were recorded in detail in the surgical notes and the injured structures were classified as strain, partial or complete tears. Since the injuries of nerve and vascular can not be fully revealed by intraoperative findings, preoperative MRI results were referenced. The exclusion criteria were (1) Revision of failed reconstructed ligaments.
(2) Periarticular tumors, infections, or congenital disorders that were found during the surgery.

After admission, a standard 1.5-Tesla MRI was performed during the acute phase of the injuries. The MRI was performed using the Turbo Spin Echo (TSE) technique, The sequence parameters were: TE (20-100 ms), TR (3000-4000 ms), slice thickness $(4.0 \mathrm{~mm})$, spacing $(0.5-1 \mathrm{~mm})$, matrix (> 256*224), and FOV $(180-230 \mathrm{~mm})$. Perioperative X-Rays and CT scans were also performed to observe whether there were periarticular fractures and other lesions. Computed tomographic angiography (CTA) was performed to identify the injuries of arteries. All patients underwent a standard physical examination of the injured knees under anesthesia, the knee laxity was quantified by the stress test, knee arthrometer, and stress $\mathrm{X}$-rays. The results were compared with the uninjured knee, and side-to-side differences were recorded. The reference standard was intraoperative findings. General information including age and gender was recorded.

\section{Evaluation of the diagnostic value of MRI}

The MR images were analyzed by two experienced musculoskeletal radiologists who were blinded to the injury patterns independently to check the presence of injuries to the ACL, PCL, MCL, LCL, PLC, and meniscus. The MRI diagnosis was performed preoperatively, and the two radiologists were blinded to the MRI findings of each other. According to the integrity of the ligaments, the injured ligaments were classified as a partial tear (ligament was ruptured but was continuous, Grade 1 or 2) or a complete tear (interruption of ligament integrity, Grade 3 ), or avulsion of the ligament endpoints. The injured ligaments and meniscus were compared with intraoperative findings that were extracted from the surgical records. Disruption of periarticular bone tissues (including femoral condyle, tibial plateau, tibial intercondylar eminence, patella, and fibula head) was defined as periarticular fractures. The exclusion criteria were bone contusion, edema of bone marrow, and bony avulsion of ligaments. The reference standard of periarticular fractures was identified through preoperative X-ray, CT scan, and intraoperative findings either from an open or arthroscopic approach. The surgeries were performed by three chief surgeons, all patients underwent a single-stage reconstruction [15]. The ACL and PCL were reconstructed with autograft gracilis and semitendinosus. For MCL/LCL, a partial tear of MCL/LCL was sutured by non-absorbable wires; bony avulsions of the insertion were fixed with a suture anchor; ruptures in the mid-substance that cannot be repaired were reconstructed with a semitendinosus autograft.

The kappa statistic was used to determine the agreement between MRI and intraoperative findings. The diagnostic value of MRI was evaluated by calculating 
the sensitivity, specificity, positive predictive value (PPV), negative predictive value (NPV), positive likelihood ratio (PLR), negative likelihood ratio (NLR), accuracy, and kappa value. The accuracy was calculated by the (true positive + true negative)/( true positive + true negative + false positive + false negative). The sensitivity, specificity, and accuracy were defined as high (accuracy $\geq 85 \%)$, moderate $(65 \% \leq$ accuray $<85 \%)$, fair $(50 \% \leq$ accuracy<65\%), low $(<50 \%)$ [8]. The classification of MLKIs was based on the criteria that were described by Schenck et al. [16], both for the MRI results and the intraoperative findings. The results were compared and the agreement between them was evaluated by calculating the kappa value. The agreement was defined as good (kappa value $\geq 0.75)$; moderate $(0.4 \leq k a p p a$ value $<0.75)$, poor (kappa value $<0.4)$; meaningless (kappa value $\leq 0)$ [17].

\section{Statistical analysis}

SPSS 25.0 (IBM Corp., Armonk, NY, USA) was used for statistical analyses. The normality of the quantitative data was checked by the Kolmogorov-Smirnov test. Normally distributed quantitative data were expressed by Mean \pm Standard Deviation $(S D)$, non-normal distributed quantitative data were presented as the interquartile range $(I Q R)$. Descriptive data were presented as numbers and percentages. The Kappa statistic was used to evaluate the agreement between the MRI and intraoperative findings, and the inter-rater reliability between the two radiologists for the MRI findings. Statistical significance was defined as $P<0.05$.

\section{Results}

\section{Study cohort}

A total of 173 patients that were diagnosed with MLKIs were included for screening, 53 patients were excluded according to the criteria, the MRI results were not available in 23 patients. Finally, 97 patients (97 injured knees) were included for analysis. There were 69 males (71.1\%) and 28 females $(28.9 \%)$, the mean age was $41.3( \pm 1.7)$ years. The combination of reconstructed ligaments and injured structures are described in Table 1. The MLKIs were classified as $20 \mathrm{KD}$-I (20.6\%), $4 \mathrm{KD}$-II (4.1\%), 47 KD-IIIM (48.5\%), 5 KD-IIIL (5.2\%), and 21 KD-V (21.6\%) according to intraoperative findings (Table 2).

\section{Diagnostic value of MRI}

The overall agreement across all MRI results between the two radiologists was good ( $k a p p a=0.83, P<0.001)$. MRI was found to have high sensitivity (90.7\%) and moderate specificity (63.6\%) in the diagnosis of injuries to the ACL; high sensitivity $(90.4 \%)$ and moderate specificity (50\%) in the diagnosis of injuries to the PCL; moderate sensitivity (79.1\%) and low specificity (46.7\%) in the diagnosis of injuries to the MCL; fair sensitivity $(55.6 \%)$ and moderate specificity $(68.4 \%)$ in the diagnosis of injuries to the LCL; fair sensitivity (61.5\%) and low specificity (39.4\%) in the diagnosis of injuries to the meniscus. The accuracy was good for ACL injuries (87.6\%) and PCL injuries (84.5\%), moderate for MCL injuries (69.1\%) and LCL injuries (66.9\%), low for the meniscus injuries (45.4\%). The agreement between MRI results and intraoperative findings was moderate in the ACL injuries (kappa $=0.47)$ and PCL injuries (kappa $=0.39$ ), poor in the MCL injuries $(k a p p a=0.26)$ and LCL injuries $(k a p p a=0.18)$, and meaningless in tears of the meniscus (Table 3). Only one of the 9 injured PLC was revealed by preoperative MRI, so the sensitivity and specificity cannot be calculated. The preoperative MR images of the injured ligaments are shown in Fig. 1.

Of the 97 combined injuries, 7 could not be classified by preoperative MRI findings. The classification based on MRI results and intraoperative findings was consistent in 51 patients (52.6\%), of them, there were $13 \mathrm{KD}-\mathrm{I}$ (25.4\%), 3 KD-II (5.9\%), 22 KD-IIIM (43.1\%), 1 KD-IIIL (1.9\%), and $12 \mathrm{KD}-\mathrm{V}$ (23.5\%). The kappa statistics showed that MRI has a moderate agreement with intraoperative findings in classifying KD-V (kappa value $=0.57$ ), poor agreement in the KD-I (kappa value $=0.39)$ and KD-IIIM ( kappa value $=0.31$ ), while meaningless in the KD-II and KD-IIIL (kappa value $<0$ ). The overall agreement

Table 1 The definition of classification and number of patients classified in each category using intra-operative findings

\begin{tabular}{|c|c|c|}
\hline Grade & Definition & $\begin{array}{l}\text { Number } \\
\text { of } \\
\text { patients }\end{array}$ \\
\hline KD-I & Two ligaments ruptured, one cruciate ligament and one collateral ligament, $A C L / P C L+M C L / L C L / P L C$ & 20 \\
\hline KD-\| & Two ligaments ruptured, both cruciate ligaments, $A C L+P C L$ & 4 \\
\hline KD-III & $\begin{array}{l}\text { Three ligaments ruptured, both cruciate ligaments, and one collateral ligament, } A C L+P C L+M C L(K D-I I I M) \text {, } \\
A C L+P C L+L C L / P L C(K D-\| I L)\end{array}$ & 52 \\
\hline KD-IV & Both two cruciate ligaments and two collateral ligaments ruptured, $A C L+P C L+M C L+L C L / P L C$ & 0 \\
\hline KD-V & Any KD classifications that were accompanied by periarticular fractures or knee dislocations & 21 \\
\hline
\end{tabular}


Table 2 Demographic characteristics and the combined injuries of the study cohort

\begin{tabular}{|c|c|c|c|c|c|c|c|c|}
\hline Reconstructed ligaments & $\mathrm{n}$ & Age (year, mean $\pm S D$ ) & Male/Female (n) & $\begin{array}{l}\text { Meniscus } \\
\text { injury (n) }\end{array}$ & $\begin{array}{l}\text { Artery } \\
\text { injury } \\
\text { (n) }\end{array}$ & $\begin{array}{l}\text { Nerves } \\
\text { injury (n) }\end{array}$ & $\begin{array}{l}\text { Patellar } \\
\text { dislocation } \\
\text { (n) }\end{array}$ & $\begin{array}{l}\text { Periarticular } \\
\text { fracture }(n)\end{array}$ \\
\hline $\mathrm{ACL}+\mathrm{MCL}$ & 15 & $38.2 \pm 8.5$ & $9 / 6$ & 5 & 1 & - & 2 & 5 \\
\hline$A C L+L C L$ & 1 & 45 & $0 / 1$ & - & - & - & - & - \\
\hline $\mathrm{ACL}+\mathrm{PLC}^{*}$ & 2 & $39.5 \pm 2.1$ & $1 / 1$ & - & - & - & - & 1 \\
\hline$P C L+M C L$ & 3 & $43.7 \pm 9.1$ & $1 / 2$ & - & - & - & - & - \\
\hline$P C L+L C L$ & 2 & $48.5 \pm 3.5$ & $2 / 0$ & - & - & - & - & 1 \\
\hline$P C L+P L C$ & 6 & $41.5 \pm 16.1$ & $6 / 0$ & 1 & - & - & - & 1 \\
\hline $\mathrm{ACL}+\mathrm{PCL}$ & 8 & $41.7 \pm 14.0$ & $6 / 2$ & 7 & 2 & 1 & 1 & 3 \\
\hline$A C L+P C L+M C L$ & 52 & $43.9 \pm 11.9$ & $37 / 15$ & 12 & - & 2 & 7 & 8 \\
\hline$A C L+P C L+L C L$ & 7 & $41.7 \pm 16.0$ & $7 / 0$ & 3 & 1 & 1 & - & 2 \\
\hline$A C L+P C L+P L C$ & 1 & 57 & $1 / 0$ & - & 1 & - & - & - \\
\hline Entire cohort & 97 & $42.6 \pm 11.8$ & $70 / 27$ & 28 & 5 & 4 & 10 & 21 \\
\hline
\end{tabular}

$S D$ standard deviation

- The number was zero

Table 3 The diagnostic value of MRI in the MLKIs

\begin{tabular}{|c|c|c|c|c|c|c|c|c|c|}
\hline Structure & Sensitivity (\%) & Specificity (\%) & PPV (\%) & NPV (\%) & PLR & NLR & Accuracy (\%) & Kappa value ${ }^{a}$ & $P$ value $^{\mathrm{b}}$ \\
\hline$A C L$ & 90.7 & 63.6 & 95.1 & 46.7 & 2.5 & 0.2 & 87.6 & 0.47 & $<0.001$ \\
\hline$P C L$ & 90.4 & 50 & 91.5 & 46.7 & 1.8 & 0.2 & 84.5 & 0.39 & $<0.001$ \\
\hline $\mathrm{MCL}$ & 79.1 & 46.7 & 76.8 & 50 & 1.5 & 0.5 & 69.1 & 0.26 & 0.010 \\
\hline LCL & 55.6 & 68.4 & 28.6 & 87.1 & 1.8 & 0.7 & 65.9 & 0.18 & 0.057 \\
\hline Meniscus & 61.5 & 39.4 & 27.1 & 73.7 & 1.0 & 0.9 & 45.4 & 0.01 & 0.931 \\
\hline
\end{tabular}

PPV positive predictive value; NPV negative predict value; PLR positive likelihood ratio; NLR negative likelihood ratio

${ }^{a}$ The agreement was good (kappa value $\left.\geq 0.75\right)$; moderate $(0.4 \leq$ kappa value $<0.75)$; poor (kappa value $\left.<0.4\right)$; meaningless $($ kappa value $<0)$

${ }^{b}$ If $p<0.05$, the agreement is significant

in classifying MLKIs was poor (kappa value $=0.23$ ) (Table 4).

\section{Discussion}

The present study found that the accuracy of MRI was good for detecting cruciate ligament injuries, moderate for collateral ligament injuries, low for meniscus injuries. We reviewed the literature and did not find research that investigated the value of MRI in classifying MLKIs. Though MRI performs better in classifying KD-V MLKIs, the overall agreement with intraoperative findings was poor. In short, MRI helps early detection of MLKIs, however, it has limited value in classifying the MLKIs preoperatively. The management of MLKIs should be based on comprehensive assessment including preoperative imaging, physical exam, and intraoperative exploration.

The diagnostic value of MRI in detecting multi-ligament injuries was evaluated by comparing the MRI results with clinical examination and/or intraoperative findings, but the results differ a lot [9-11, 14]. In most studies, MRI was reliable in detecting ligament injuries, however, in terms of meniscus and PLC, the conclusions were controversial $[7,8]$. In the present study, the accuracy of MRI in detecting cruciate ligaments was consistent with previous studies. The accuracy was demonstrated moderate in detecting injuries to collateral ligaments, which was rarely reported in MLKIs. Research has suggested that oblique coronal and oblique sagittal MRI, which was parallel to the long axis of the ACL, improved the accuracy of the diagnosis of an ACL tear and the grading of ACL injury [18-20]. However, the application of oblique MRI in multiple ligaments injuries is limited. The evaluation of the value of MRI in diagnosing MLKIs should also consider the interpretation of MRI results [21]. Since the MLKIs were complex injuries, the accuracy of MRI for diagnosing isolated ligament injuries was not comparable with that of the multi-ligament injuries. In short, this study concluded that MRI was valuable for the early diagnosis of MLKIs.

The value of MRI in classifying MLKIs according to Schenck classification was explored in this study. We find that MRI has a moderate agreement in classifying KD-V, 


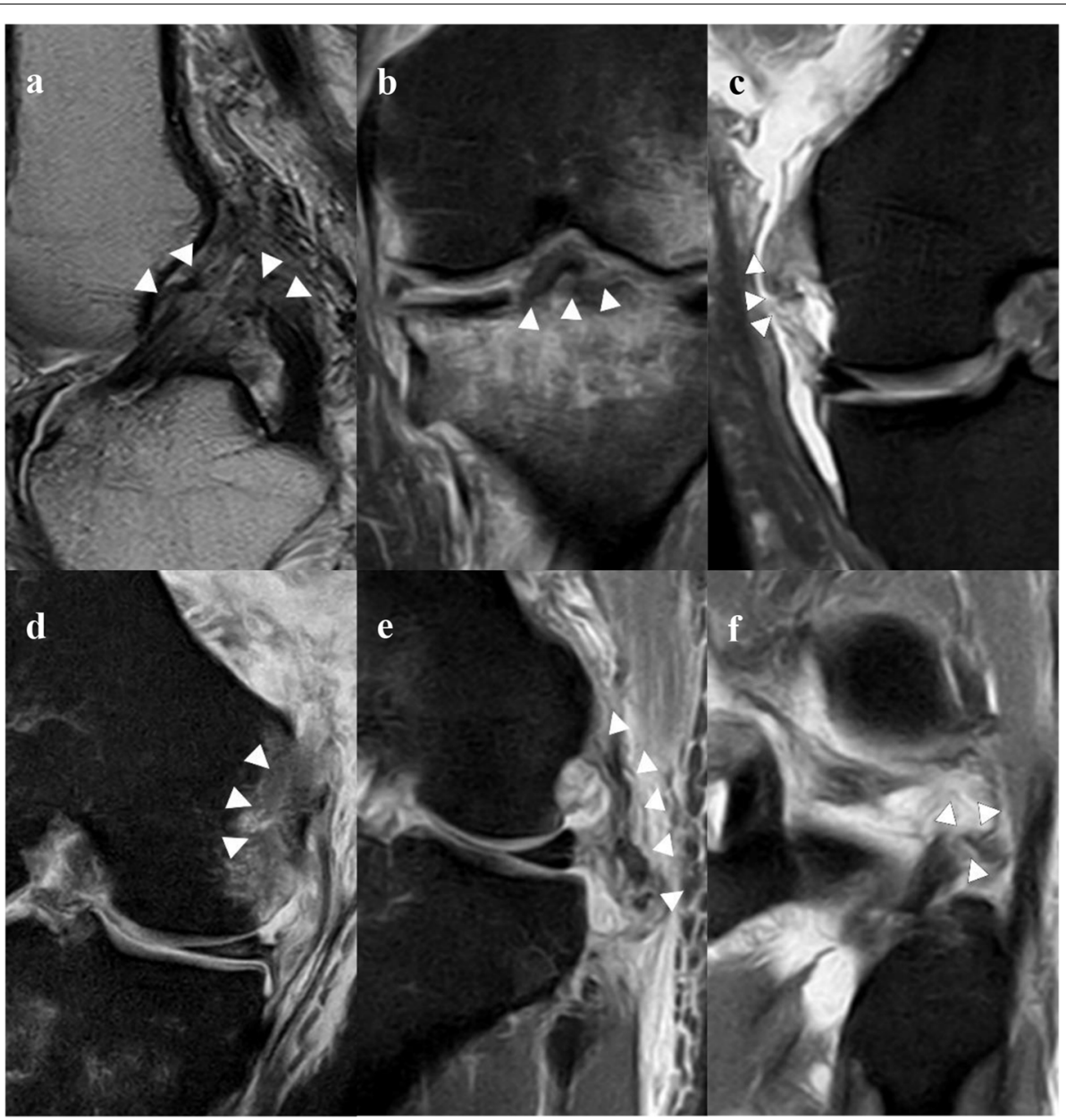

Fig. 1 Preoperative MRI of the multiple ligaments knee injuries/knee dislocations; a The ruptured ACL and PCL; b Avulsion fracture at the ACL insertion in the tibial intercondylar eminence; $\mathbf{c}$ Rupture of $\mathrm{MCL}$ in the mid-substance; $\mathbf{d}$ Avulsion of $\mathrm{MCL}$ at the insertion of the femoral condyle; $\mathbf{e}$ The ruptured $L C L$ in the mid-substance; $\mathbf{f}$ The ruptured popliteal tendon

Table 4 The value of MRI in classifying MLKIs according to Schenck classification (n)

\begin{tabular}{|c|c|c|c|c|c|c|c|c|c|c|}
\hline \multirow{2}{*}{$\begin{array}{l}\text { Intraoperative } \\
\text { classification (n) }\end{array}$} & \multicolumn{7}{|c|}{ MRI classification ( $\mathrm{n}$ ) } & \multirow[t]{2}{*}{ Total (n) } & \multirow[t]{2}{*}{ Kappa value $^{\#}$} & \multirow[t]{2}{*}{$P$ value ${ }^{8}$} \\
\hline & 1 & II & IIIM & IIIL & IV & $\mathbf{v}$ & $\mathrm{NA}^{*}$ & & & \\
\hline I & 13 & - & 1 & 1 & 4 & - & 1 & 20 & 0.39 & 0.019 \\
\hline$\|$ & 2 & 3 & 2 & 1 & 2 & - & - & 10 & -0.07 & 0.619 \\
\hline IIIM & 1 & 8 & 22 & 4 & 6 & - & 3 & 44 & 0.31 & 0.003 \\
\hline IIIL & - & 4 & 1 & 1 & 1 & - & 1 & 8 & -0.21 & 0.262 \\
\hline IV & - & - & - & - & - & - & - & - & - & - \\
\hline V & - & - & - & 1 & - & 12 & 2 & 15 & 0.57 & 0.007 \\
\hline Total & 16 & 15 & 26 & 8 & 13 & 12 & 7 & 97 & 0.23 & 0.001 \\
\hline
\end{tabular}

\footnotetext{
*NA Cannot be classified by Schenck classification
}

\#The agreement was good (kappa value $\geq 0.75)$; moderate $(0.4 \leq$ kappa value $<0.75)$; poor (kappa value $<0.4)$; meaningless $($ kappa value $<0)$

\& If $p<0.05$, the agreement is significant

- The number was zero 
poor agreement in classifying KD-I and KD-IIIM, meaningless in KD-II and KD- IIIL. We speculate that the meaningless agreements in the KD-II and KD-IIIL were due to the small numbers of those two injuries, the diagnostic value cannot be reflected well. Though inferior to the CT scan, the present study revealed that MRI helps detect periarticular fractures (moderate consistency with intraoperative findings in classifying KD-V). Besides, we found that MRI has high sensitivity in detecting ACL and PCL injuries, but the overall agreement was poor compared to intraoperative findings. The results were not surprising because the MLKIs are complex injuries, a precise MRI-based classification is challengeable. Though the sensitivity and specificity in this study differ from previous studies, we concluded MRI has limited value in classifying MLKIs preoperatively, the management of MLKIs should be based on a comprehensive evaluation, including physical exam, combined X-Rays, CT, and mechanisms of injuries until intraoperative evidence was obtained.

In the present study, only one of the PLC injuries was revealed by preoperative MRI, suggesting a limited value of MRI in detecting PLC injuries, there were no falsepositive cases, the sensitivity and specificity were not calculated because the number of samples was small. In fact, few studies have reported the results of PLC reconstruction because of the low incidence rate. Derby et al. [8] investigated the value of MRI in detecting the PLC for patients with knee dislocations, including LCL (76\% accuracy, $100 \%$ sensitivity, $67 \%$ specificity) and iliotibial tract (89\% accuracy, $97 \%$ specificity). In the present study, only 9 cases were diagnosed with PLC injuries according to the intraoperative findings. Precise detection of PLC injuries using MRI is challenging. The value of MRI in detecting PLC injuries remains unknown, the diagnosis should be based on clinical examination under anesthesia and intraoperative findings.

The present study has some limitations. First, this is a retrospective analysis with a small number of samples, there was a lack of systematic methods used to include/ exclude patients, thus inherent bias can not be avoided. Second, there was a variation of the incidence of injury patterns between groups, the intraoperative findings were considered as gold standard during the statistical analysis, which may lead to bias. Third, only a $1.5 \mathrm{~T}$ MRI magnet was used for scanning and the severity of the injured ligaments was not graded using the MR images, partial and complete tears were not divided into subgroups and evaluated. Fourth, the number of PLC injuries was small and only one of them was successfully revealed by the MRI, thus the accuracy of MRI in detecting PLC injuries can not be evaluated. Furthermore, MRI results were not compared with clinical examination.
Future studies should be based on larger samples and a more specific evaluation system.

\section{Conclusions}

This study revealed that MRI has high sensitivity but moderate specificity in detecting ACL and PCL injuries, and a fair agreement in classifying KD-V MLKIs. In short, MRI was found to have limited value in classifying MLKIs. Therefore, the management of MLKIs should be based on intraoperative findings, physical examinations, and comprehensive imaging results.

\section{Acknowledgements \\ None \\ Authors' contributions \\ Haifeng Yuan design the study and censored the manuscript. Xusheng Li col- lected the data and drafted the manuscript. Qian Hou collected the data and performed the statistical analysis. Xiaobing Ma, Xuehua Zhan, and Long Chang analyzed the MRI results. All authors read and approved the final manuscript. \\ Funding \\ This study was funded by The Application of Gene X-pert MTB/RIF Technol- ogy in the Diagnosis of Spinal Tuberculosis (2019-NW-005); Study on the mechanism of MTHFD2 induced degeneration of lumbar intervertebral disc (2020AAC03393).}

\section{Availability of data and materials}

All data generated or analyzed during this study are included in this published article.

\section{Declarations}

Ethics approval and consent to participate

This study was conducted in accordance with the ethical guidelines of the Declaration of Helsinki. This retrospective study was approved by the Research Ethics Committee of the General Hospital of Ningxia Medical University. Informed consent was obtained from all patients for this study, all patients agreed to participate in the study.

\section{Consent for publication}

All patients agreed to have their data published.

\section{Competing interests}

The authors declare that there is no conflict of competing financial and nonfinancial interests.

\section{Author details \\ ${ }^{1}$ Department of Spinal Orthopaedics, General Hospital of Ningxia Medical University, No. of 804, Shengli Street, Yinchuan 750004, China. ${ }^{2}$ Department of Radiology, General Hospital of Ningxia Medical University, No. of 804, Shengli Street, Yinchuan 750004, China.}

Received: 24 May 2021 Accepted: 20 December 2021

Published online: 13 January 2022

\section{References}

1. Fanelli GC. Multiple Ligament Injured Knee: Initial Assessment and Treatment. Clin Sports Med. 2019;38(2):193-8.

2. Lachman JR, Rehman S, Pipitone PS. Traumatic Knee Dislocations: Evaluation, Management, and Surgical Treatment. Orthop Clin North Am. 2015:46(4):479-93. 
3. Hua X, Tao H, Fang W, Tang J. Single-stage in situ suture repair of multipleligament knee injury: a retrospective study of 17 patients (18 knees). BMC Musculoskelet Disord. 2016;17:41.

4. Azar FM, Brandt JC, Miller RH 3rd, Phillips BB. Ultra-low-velocity knee dislocations. Am J Sports Med. 2011;39(10):2170-4.

5. Peskun CJ, Levy BA, Fanelli GC, Stannard JP, Stuart MJ, Macdonald PB, et al. Diagnosis and management of knee dislocations. Phys Sportsmed. 2010;38(4):101-11.

6. Hirschmann MT, Zimmermann N, Rychen T, Candrian C, Hudetz D, Lorez $L G$, et al. Clinical and radiological outcomes after management of traumatic knee dislocation by open single stage complete reconstruction/ repair. BMC Musculoskelet Disord. 2010;11:102.

7. Reddy CG, Amrami KK, Howe BM, Spinner RJ. Combined common peroneal and tibial nerve injury after knee dislocation: one injury or two? An MRI-clinical correlation. Neurosurg Focus. 2015;39(3):E8.

8. Derby E, Imrecke J, Henckel J, Hirschmann A, Amsler F, Hirschmann MT. How sensitive and specific is 1.5 Tesla MRI for diagnosing injuries in patients with knee dislocation? Knee Surgery, Sports Traumatology, Arthroscopy. 2017;25(2):517-23.

9. Twaddle BC, Hunter JC, Chapman JR, Simonian PT, Escobedo EM. MRI in acute knee dislocation. A prospective study of clinical, MRI, and surgical findings. J Bone Joint Surg Br. 1996;78(4):573-9.

10. Munshi M, Davidson M, Macdonald PB, Froese W, Sutherland K. The efficacy of magnetic resonance imaging in acute knee injuries. Clin J Sport Med. 2000;10(1):34-9.

11. Halinen J, Koivikko M, Lindahl J, Hirvensalo E. The efficacy of magnetic resonance imaging in acute multi-ligament injuries. Int Orthop. 2009;33(6):1733-8.

12. Kosy JD, Matteliano L, Rastogi A, Pearce D, Whelan DB. Meniscal root tears occur frequently in multi-ligament knee injury and can be predicted by associated MRI injury patterns. Knee Surg Sports Traumatol Arthrosc. 2018;26(12):3731-7.

13. Barbier O, Galaud B, Descamps S, Boisrenoult P, Leray E, Lustig S, et al. Relevancy and reproducibility of magnetic resonance imaging (MRI) interpretation in multiple-ligament injuries and dislocations of the knee. Orthop Traumatol Surg Res. 2013;99(3):305-11.

14. Lonner JH, Dupuy DE, Siliski JM. Comparison of magnetic resonance imaging with operative findings in acute traumatic dislocations of the adult knee. J Orthop Trauma. 2000;14(3):183-6.

15. Laprade RF, Chahla J, Dephillipo NN, Cram T, Kennedy MI, Cinque $M$, et al. Single-Stage Multiple-Ligament Knee Reconstructions for Sports-Related Injuries: Outcomes in 194 Patients. Am J Sports Med 2019:47(11):2563-71.

16. Schenck R. Classification of knee dislocations. Operat Techniq Sports Med. 2003;11(3):193-8.

17. Vaccaro AR, Schroeder GD, Divi SN, Kepler CK, Kleweno CP, Krieg JC, et al. Description and Reliability of the AOSpine Sacral Classification System. J Bone Joint Surg Am. 2020;102(16):1454-63.

18. Kosaka M, Nakase J, Toratani T, Ohashi Y, Kitaoka K, Yamada H, et al. Oblique coronal and oblique sagittal MRI for diagnosis of anterior cruciate ligament tears and evaluation of anterior cruciate ligament remnant tissue. Knee. 2014;21(1):54-7.

19. Ghasem Hanafi M, Momen Gharibvand M, Jaffari Gharibvand R, Sadoni H. Diagnostic Value of Oblique Coronal and Oblique Sagittal Magnetic Resonance Imaging (MRI) in Diagnosis of Anterior Cruciate Ligament (ACL) Tears. J Med Life. 2018;11(4):281-5.

20. Hong SH, Choi JY, Lee GK, Choi JA, Chung HW, Kang HS. Grading of anterior cruciate ligament injury. Diagnostic efficacy of oblique coronal magnetic resonance imaging of the knee. J Comput Assist Tomogr. 2003;27(5):814-9.

21. Zhai G, Ding C, Cicuttini F, Jones G. Optimal sampling of MRI slices for the assessment of knee cartilage volume for cross-sectional and longitudinal studies. BMC Musculoskelet Disord. 2005;6:10.

\section{Publisher's Note}

Springer Nature remains neutral with regard to jurisdictional claims in published maps and institutional affiliations.

Ready to submit your research? Choose BMC and benefit from:

- fast, convenient online submission

- thorough peer review by experienced researchers in your field

- rapid publication on acceptance

- support for research data, including large and complex data types

- gold Open Access which fosters wider collaboration and increased citations

- maximum visibility for your research: over 100M website views per year

At BMC, research is always in progress.

Learn more biomedcentral.com/submissions 